\title{
Implication of pharmacists in Cluj County in managing the COVID-19 pandemic
}

\author{
Elena DINTE ${ }^{1,2}$, Teodora ZEHAN¹, George Florin MICLAUS², Niculina Ileana SANDOR², \\ Daniela VAIDA², Simona VOSTINARU² \\ ${ }^{1}$ Department of Pharmaceutical Technology and Biopharmaceutics, Faculty of Pharmacy, \\ "Iuliu Hatieganu" University of Medicine and Pharmacy, Cluj-Napoca, Romania \\ ${ }^{2}$ College of Pharmacists, Cluj County, Romania
}

\begin{abstract}
Objectives. To highlight the involvement of pharmacists working in community pharmacies in the management of the COVID-19 pandemic, to identify the main services provided by pharmacists in order to sustain pharmaceutical care and the main actions taken to minimize the encountered difficulties during the March 16th 2020 - September 15th 2020 timeframe.

Material and methods. A questionnaire was elaborated and then distributed to community pharmacies in Cluj County. Based on the results obtained after the completion of the questionnaire, an evaluation of the following factors could be made: pharmacy opening hours, staff plan and the shortage of supplies and personal protective equipment against the Coronavirus disease.

Results. The activity in a total of 246 community pharmacies (80.92\% of the total number of community pharmacies in Cluj County) was analyzed, of which 134 community pharmacies (54.47\% of the number of analyzed ones) adjusted their opening hours, most frequently dependent on the requirements and conditions generated by the state of emergency (in $73.88 \%$ of the number of analyzed cases), some of which chose to adjust the staff plan as well. The most important matter that affected the activity of pharmacists during the pandemic was the shortage of personal protective equipment (such as face masks and gloves), as well as the shortage of pharmaceutical grade ethanol, intended for the preparation of antiseptic and disinfectant solutions (sanitizers).

Conclusions. The pharmacists have shown an active involvement in maintaining the pharmaceutical service and the public health of the population during the COVID-19 pandemic as a part of the frontline healthcare professionals. They were able to elaborate new strategies adapted to the requirements generated by the community and to the restrictions generated by the state of emergency in order to fulfil their duty. During this period of time, the importance of preparation activity in pharmacy was strongly emphasized, the most frequent problem encountered being the difficult supply with personal protective materials.
\end{abstract}

Keywords: COVID-19, pandemic, state of emergency, role of pharmacist, community pharmacy

\section{INTRODUCTION}

The Coronavirus Disease 2019

(COVID-19) is an infection caused by the Severe Acute Respiratory Syndrome Coronavirus 2 (SARSCoV-2), which made its first apparition in December 2019, in Wuhan (China), with a high rate of infectiousness $(1,2)$. 
Because of its rapid spread across the world, the World Health Organization (WHO) declared COVID-19, on March 11th 2020, a global pandemic. Taking this into consideration and observing the evolution of the epidemiological risk worldwide, the President of Romania declared the state of emergency on the territory of our country, on March 16th 2020, by Presidential Decree no. 195/2020 (3). The COVID-19 infection is characterized by fever, cough, difficulty breathing or shortness of breath (in the more severe cases of infection), tiredness and headache. Most of the patients who contact the disease suffer from mild to moderate symptoms, but more drastic cases can appear, and patients' condition can progress to serious illness and even death. Up to present, there is no treatment with proven effectiveness against COVID-19 disease, and before a vaccine is going to be developed, the population must follow the special guidelines established by the World Health Organization (WHO) which are meant to limit the spread of the virus in the community: strict hand hygiene, mask wearing and social distancing $(1,2)$. This situation represented a challenge for all the healthcare professionals in our country, pharmacists included, who were able to contribute to the critical situation with all their resources and abilities. Based on their training, pharmacists have made an important contribution to the management of the state of emergency, supporting the national authorities by their intervention in the healthcare system. They provided pharmaceutical care, ensuring the continuity of access to medicine destined for the patients with acute or chronic diseases, as well as providing reliable information regarding the Coronavirus infection and ways to protect against it, detect and manage such an infection. Pharmacists were also able to prepare antiseptic and disinfectant solutions in the community pharmacies where this option was available, solutions which were of a very high necessity during the shortage of sanitizers and other antiseptic supplies produced by pharmaceutical companies which were overwhelmed by the high demand of these goods all over the world.

This paper aims to highlight the actions taken by pharmacists in Cluj County in order to optimally manage the crisis caused by the COVID-19 pandemic and to identify the issues that had a significant impact on their activity in the March 16th 2020 - September 15th 2020 timeframe.

\section{MATERIALS AND METHODS}

The objective of the study is represented by a retrospective analysis of certain factors that influenced the activity of pharmacists in Cluj County, designed to highlight the implication of pharmacists in the healthcare system from the establishment of the state of emergency in the country until now (from March 16th 2020 to September 15th 2020). A questionnaire was elaborated and then distributed to community pharmacies in Cluj County, and based on the answers, aspects regarding the following factors were analyzed: pharmacy opening hours, staff plan and the difficulty of acquiring personal protective equipment against COVID-19 infection. The questionnaire was 


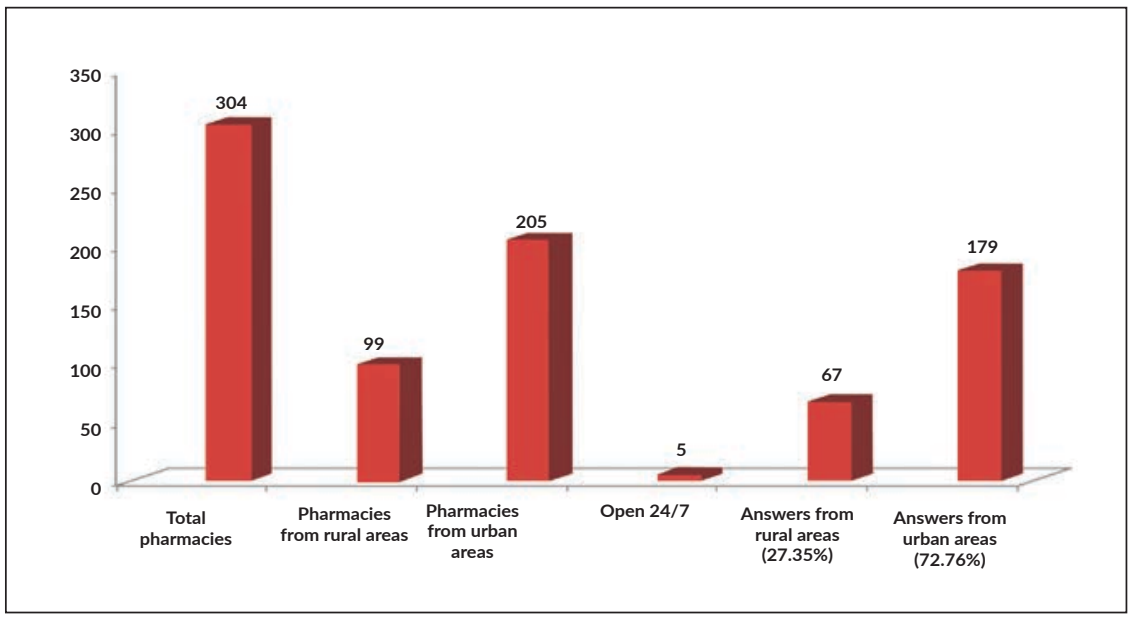

FIGURE 1. The distribution of existing community pharmacies in the Cluj County, next to the distribution of the ones which participated in the study, spread in rural and urban areas

completed by the head pharmacist, and the questions referred to the activity in the community pharmacy during the COVID-19 pandemic, without requesting any personal data from the respondent.

\section{RESULTS AND DISCUSSIONS}

The questionnaire was delivered to all the community pharmacies in Cluj County, meaning 304 pharmacies, of which 99 from the rural area, and the rest of 205, from the urban environment. A number of 246 responded, of which 67 from the rural area and 179 from the urban environment (representing a percent of $27.35 \%$ - rural, and $72.76 \%$ - urban, percentages based on the total number of respondent pharmacies) (Fig. 1).

\section{Ensuring the continuity of population access to medication} After the state of emergency was declared, pharmacists in Romania continued to ensure the population access to the medicine and, just as all the others healthcare professionals, they remained in the front line. A specific set of procedures was elaborated and implemented, regarding medication dispensing, sanitizing the space in the pharmacy and complying with the rules imposed by authorities in order to prevent the spread of the SARS-CoV-2 virus.

By analyzing the activity of the pharmacies in the Cluj County, it can be highlighted that a number of 134 of pharmacies (44\% of the total number of pharmacies in the county) modified their operating schedule, which was reduced to a smaller number of opening hours, and in the case of two pharmacies with both day and night operating hours, the night shift was canceled during the state of emergency; the reduction of working hours was justified in $73.88 \%$ of the cases by adapting the operating schedule to the new conditions imposed by the state of emergency (decreased visits to the pharmacy related to the restrictions of movement and the reduced patient demands for certain timeframes). Some of the chief pharmacists justified the changes in operating hours by the difficult conditions in which pharmacists had to carry out their work and the need to take several 
additional measures to prevent the contamination of staff - this is the case in $57.46 \%$ of the total number of pharmacies (adopted changes: introduction of a break of 30-60 minutes between working teams, in order to disinfect the pharmacy and to limit contact between teams, shorter work shifts), whereas just a small percentage of $8.95 \%$ of the pharmacies made this decision because of insufficient staff. After the cessation of the state of emergency, $70 \%$ of the pharmacies which, in the beginning of the pandemic modified their operating schedule, have returned, up to present, to the initial opening hours.

Besides modifying the operating schedule, the respondent pharmacies reported 26 cases in which, in order to ensure a balanced functioning of the pharmacy, they resorted to a change in the staff plan as well: transfer of pharmacists between shifts or between pharmacies, 18 reported resignations (various reasons), 6 cases in which pharmacists became technically unemployed and 2 cases of COVID-19 infection during the analyzed period (Fig. 3). There was no quantification of other situations which influenced the staff plan and which did not have a direct relation to the COVID-19 pandemic, namely rest leaves and maternity leaves.

\section{Implementation of COVID-19} prevention and control procedures In order to prevent the spread of the SARS-CoV-2 virus, pharmacists have implemented a series of measures to adapt pharmacy activities to the new restrictions imposed by the pandemic, in order to protect patients and staff, such as:

- measures regarding the adaptation of the medicine disposal process to patients: limiting the access of the patients inside the pharmacy and dispensing at the window, dispensing medicines on the basis of prescriptions sent electronically, observance of specific rules for storing and archiving documents; - specific measures regarding the protection against the SARS-CoV-2 virus: effective disinfection of the pharmacy space and work surfaces, implementing the use of protective

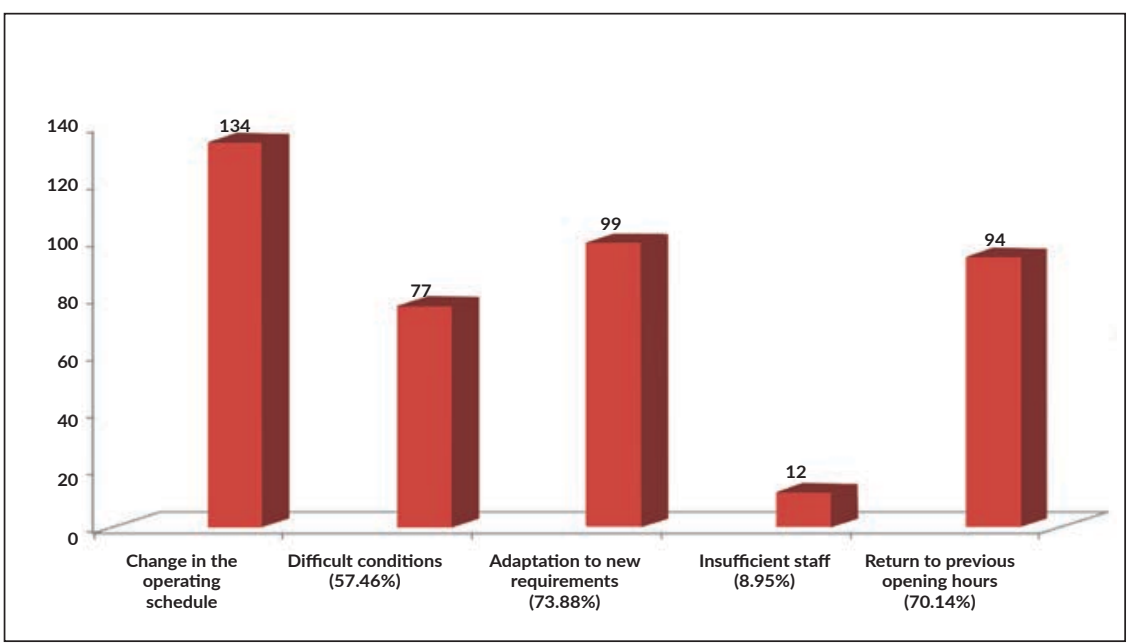

FIGURE 2. The factors that influenced the change in the operating schedule of community pharmacies in Cluj County, during the COVID-19 pandemic 
screens to limit the contact between the pharmacist and the patient, use of personal protective equipment (gloves, face masks, visors), rigorous asepsis of the hands

One of the problems that was reported in the mass-media, right at the beginning of the pandemic, was represented by the difficulty of supplying personal protective equipment in the pharmacies. Insufficient stocks of face masks and gloves at specialized distributors also aggravated the activity of the pharmacist in the community pharmacy. Pharmacists in Cluj County were asked if the shortage in stocks of protective materials in pharmacies was due to lower purchases as a result of a higher price level at the distributor (which would have meant an additional financial support from the pharmacy), or the absence of said materials in the stock of suppliers. A number of 230 pharmacies (representing a percentage of $93.49 \%$ of the number of respondents) declared

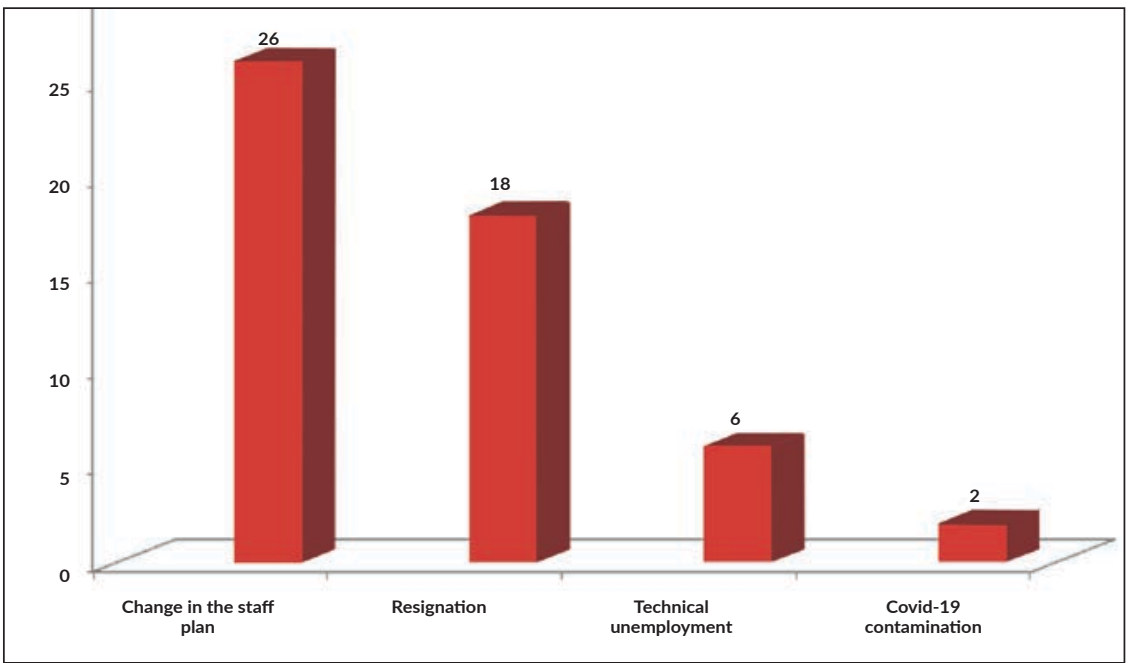

FIGURE 3. The factors that determined changes in the staff plan in community pharmacies in Cluj County, in relation to the COVID-19 pandemic

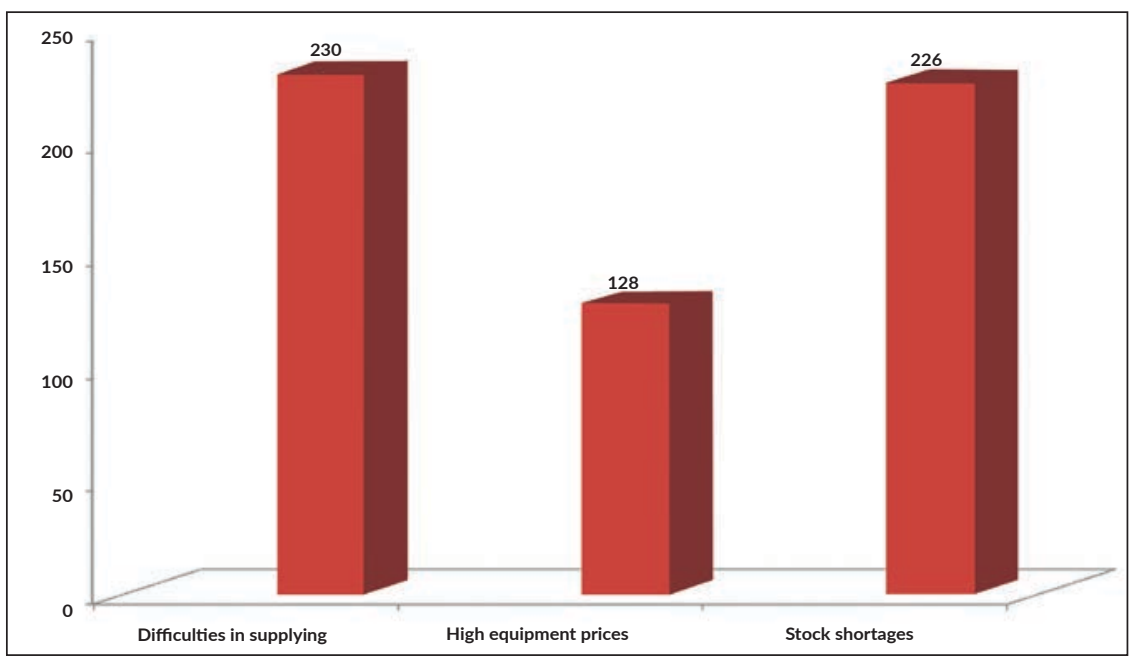

FIGURE 4. Analysis of supply difficulties with personal protective equipment encountered by community pharmacies from Cluj County, at the beginning of the COVID-19 pandemic 


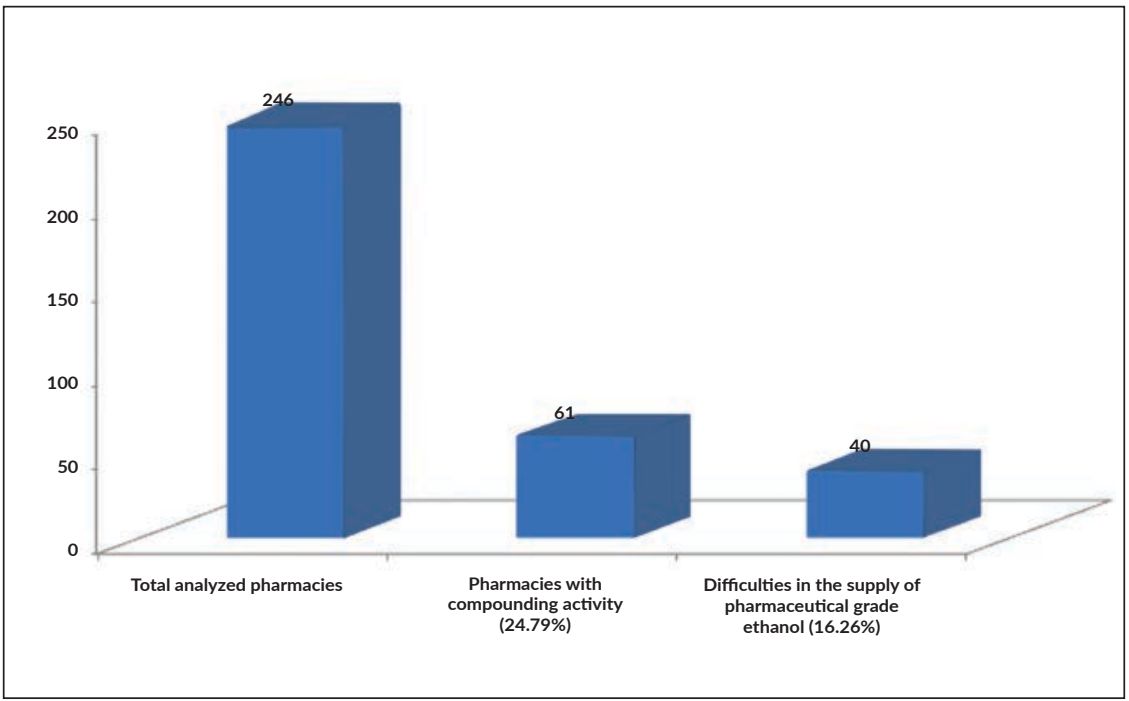

FIGURE 5. Analysis of the difficulties encountered by community pharmacies with compounding activity from Cluj County, in relation to the preparation of antiseptic and disinfectant solutions, at the beginning of the COVID-19 pandemic

that the supply was very difficult at the beginning of the pandemic, and a part of these pharmacies, meaning a number of 128 , confirmed the rise they encountered in product prices. At the same time, 226 pharmacies confirmed that the biggest impediment in supply was the absence of protective materials in the stocks of distributors. The chief pharmacists representing the respondents to the study mentioned that they purchased gloves and face masks at very high prices compared to the price ranges found in the period of time before the pandemic, in order to ensure optimal protection of pharmacy staff (Fig. 4).

\section{Preparation of antiseptic and disinfectant solutions}

Another problem that pharmacists encountered, at the beginning of the pandemic, was represented by the difficult supply with pharmaceutical grade ethanol, destined for the preparation of antiseptic and disinfectant solutions. Because there was a significant shortage of companyproduced sanitizers and disinfectant solutions, especially during the state of emergency, pharmacists in Romania have been actively involved in maintaining the public health of citizens in the community, especially by preparing these highly demanded solutions in the laboratory of the pharmacy, where it was available. This activity of solution preparation was initiated by pharmacists in other countries as well (4). The activity of medication preparation in pharmacy, regulated in Romania by several normative acts (5-8), also includes the preparation of antiseptic and disinfectant solutions and, because of the severe shortage in pharmaceutical grade ethanol especially at the beginning of the pandemic, the preparation of these solutions was limited. Of the 246 respondent pharmacies, a number of 61 pharmacies, existing both in rural and urban areas, are authorized for the compound activity, and 40 of these pharmacies 
encountered difficulties in the supply of pharmaceutical grade ethanol (figure 5). The rest of the pharmacies had, at the moment the state of emergency was established, sufficient stocks of pharmaceutical grade ethanol to prepare antiseptic and disinfectant solutions in sufficient quantity to cover the demand of the citizens in the community they serve (pharmacies in rural areas).

\section{CONCLUSIONS}

As healthcare professionals, pharmacists have been actively involved in managing the state of emergency caused by the COVID-19 pandemic. The analysis of community pharmacies in Cluj County related to the March 16th - September 15th 2020 timeframe highlighted that, in order to ensure the continuity of population access to medication and to pharmaceutical care, pharmacists had to adapt the opening hours and staff plan according to the community needs. Also, sourcing personal protective equipment, as well as sourcing prime materials meant for the preparation of the antiseptic and disinfectant solutions, were a difficult task to complete during the pandemic.

\section{Acknowledgements}

We would like to give thanks all our colleagues pharmacists in Romania, who were actively involved and served their fellows with dedication and professionalism during the difficult and unprecedented situation created by the COVID-19 pandemic. I would like to thank Mrs. Adriana Susa and Miss Stefania Gergely, for the support provided in collecting the necessary data for the elaboration of this paper.

Conflict of interest: none declared Financial support: none declared

4. 10000 litres de solution hydroalcoolique par jour: à Paris, une pharmacie monte un laboratoire en pleine rue. Accessed on September 15th 2020. Available at: https:// france3-regions.francetvinfo.fr/paris-ile-defrance/paris/10000-litres-solutionhydroalcoolique-jour-paris-pharmaciemonte-laboratoire-pleine-rue-1808704.html ?fbclid=IwAR3XyJcHPWva4eFEcdBMrTetGjA aJC1pBLNy1abR8wqVQ-Q85VQtr4z4nVE.

5. Law no. 95/2006 on Healthcare Reform, regulating the Romanian public healthcare system and establishing its fundamentals, as republished in the Official Gazette. Internet. Accessed on September 15th 2020. Available at: https://www.anm.ro/en/_/ DM/LEGI/Titlul\%20XX_MD_2016_EN.pdf.
6. Pharmacy Law no. $266 / 2008$, as republished in the Official Gazette. Internet. Accessed on September 15th 2020. Available at: http://legislatie.just.ro/Public/ DetaliiDocument/109030.

7. Order of the Minister of Health no. $75 / 2010$ for the approval of the Rules of Good Pharmaceutical Practice, published in the Official Gazette of Romania, part I, no. 91, on February 10th 2010

8. Order of the Minister of Health no. $444 / 2019$, for the approval of the Rules of establishment, organization, and operation of pharmaceutical units, published in the Official Gazette of Romania, no. 270 bis, on April 9th 2019. 